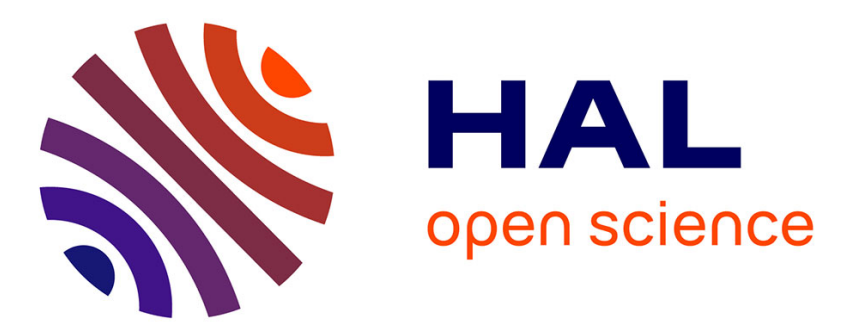

\title{
La littérature illustrée pour enfants pendant la Première Guerre mondiale et la difficile sortie de guerre de l'Allemagne
}

Bérénice Zunino

\section{- To cite this version:}

Bérénice Zunino. La littérature illustrée pour enfants pendant la Première Guerre mondiale et la difficile sortie de guerre de l'Allemagne. Les Cahiers Sirice / Irice , 2016, 17, pp.15-27. hal-03466821

\section{HAL Id: hal-03466821 \\ https://hal.science/hal-03466821}

Submitted on 2 Jan 2022

HAL is a multi-disciplinary open access archive for the deposit and dissemination of scientific research documents, whether they are published or not. The documents may come from teaching and research institutions in France or abroad, or from public or private research centers.
L'archive ouverte pluridisciplinaire HAL, est destinée au dépôt et à la diffusion de documents scientifiques de niveau recherche, publiés ou non, émanant des établissements d'enseignement et de recherche français ou étrangers, des laboratoires publics ou privés. 


\section{La littérature illustrée pour enfants pendant la Première Guerre mondiale et la difficile sortie de guerre de l'Allemagne}

Bérénice Zunino (ancienne doctorante à l'université Paris-Sorbonne / UMR 8138 et à la Freie Universität de Berlin, actuellement post-doctorante au Centre Georg Simmel / UMR 8131 EHESS/CNRS)

La question de la sortie de guerre de l'Allemagne à l'exemple de la littérature illustrée pour enfants à l'époque de la Première Guerre mondiale s'inscrit à la croisée des études sur l'enfance en guerre, sur les cultures de guerre et sur les démobilisations culturelles ${ }^{1}$. Provenant des historiographies française et britannique, ces approches ont permis d'aborder la Grande Guerre sous un angle nouveau. Pour le cas allemand, leur réception a été conditionnée par une réflexion sur les liens entre la « catastrophe originelle du XXe siècle » et la montée du national-socialisme. Pour cette raison, la question de la démobilisation culturelle a retenu le plus l'attention'2.

Outre le choc de la défaite de 1918 et des conditions jugées trop dures du traité de Versailles, les liens entre la socialisation des enfants à l'arrière entre 1914 et 1918 et la radicalisation politique d'une partie d'entre eux durant l'entre-deux-guerres ont été abordés dans plusieurs études. Elles prennent en compte les expériences de la cohorte née entre 1900 et 1910 qui vécut sa socialisation à l'arrière durant le conflit, appelée Kriegsjugendgeneration. Dans une approche générationnelle, Michael Wildt évoque dans le premier chapitre de son ouvrage consacré à la biographie des membres du Reichssicherheitshauptamt, dont plus d'un tiers était né après 1900, l'expérience marquante de ces enfants qui connurent la privation à l'arrière entre 1914 et 1918 et furent familiarisés de manière précoce avec la propagande ${ }^{3}$.

Hormis ces travaux, l'enfance durant la Première Guerre mondiale, contrairement au cas français ${ }^{4}$, a fait l'objet de peu d'études en Allemagne ${ }^{5}$. La mobilisation culturelle des enfants n'a pas été envisagée dans une approche visuelle de la culture de guerre ${ }^{6}$, notion qui en Allemagne n'a pas eu l'écho qu'elle a trouvé en France ${ }^{7}$. Dans une perspective d'histoire culturelle, nous nous proposons de revenir dans cette contribution sur l'évolution de la littérature illustrée enfantine allemande entre 1914 et 1918 et de formuler des hypothèses sur son impact potentiel à moyen terme sur les mentalités des plus jeunes. Nous souhaitons ainsi

\footnotetext{
1 Bruno Cabanes / Guillaume Piketty, «Sortir de la guerre: jalons pour une histoire en chantier», Histoire@Politique. Politique, culture, société, n 3 , nov.-déc. 2007, en ligne : www.histoire-politique.fr. [Site consulté le 26 mai 2015].

2 Gerd Krumeich, «L'impossible sortie de guerre de l'Allemagne », dans Stéphane Audoin-Rouzeau / Christophe Prochasson (dir.), Sortir de la Grande Guerre. Le monde et l'après 1918, Paris, Tallandier, 2008, p. 145-164 ; John Horne, « Démobilisations culturelles après la Grande Guerre », Revue 14-18 Aujourd'hui, $\mathrm{n}^{\circ}$ 5, 2002, p. 45-53.

3 Michael Wildt, Generation des Unbedingten. Das Führungskorps des Reichssicherheitshauptamtes, Hambourg, Hamburger Edition, 2002, p. 46-52. Les études suivantes étayent cette hypothèse : Christian Ingrao, Croire et détruire. Les intellectuels dans la machine de guerre SS, Paris, Pluriel, 2011, p. 19-48 ; Arndt Weinrich, Der Weltkrieg als Erzieher. Jugend zwischen Weimarer Republik und Nationalsozialismus, Essen, Klartext, 2013, p. 35-63.

${ }^{4}$ Stéphane Audoin-Rouzeau, La Guerre des enfants, 1914-1918, Paris, Colin, 2004 (1 ${ }^{\mathrm{e}}$ éd. 1993) ; Manon Pignot, Allons enfants de la Patrie. Génération Grande Guerre, Paris, Seuil, 2012.

5 Marieluise Christadler, Kriegserziehung im Jugendbuch. Literarische Mobilmachung in Deutschland und Frankreich vor 1914, Francfort/Main, Haag + Herchen, 1978 ; Andrew Donson, Youth in the fatherless land: war pedagogy, nationalism, and authority in Germany, 1914-1918, Cambridge, Harvard University Press, 2010.

${ }^{6}$ Philippe Kaenel / François Valloton (dir.), Les images en guerre (1914-1945), Lausanne, Antipodes, 2008.

${ }^{7}$ Pour un exposé détaillé, voir : Arnd Bauerkämper / Élise Julien, «Einleitung : Durchhalten ! Kriegskulturen und Handlungspraktiken im Ersten Weltkrieg », dans Id. (dir.), Durchhalten! Krieg und Gesellschaft im Vergleich 1914-1918, Göttingen, Vandenhoeck \& Ruprecht, 2010, p. 7-28.
} 
nuancer à l'exemple de la littérature illustrée pour enfants l'hypothèse selon laquelle la démobilisation culturelle fut peu opérante pour le cas allemand.

Bien qu'il soit malaisé de mesurer l'effet de cette littérature, les stéréotypes qu'elle véhiculait, de par leur récurrence et leur force émotionnelle, étaient susceptibles de structurer les schémas mentaux des jeunes gens et, une fois ancrés dans l'identité collective, d'avoir une influence à long terme. Les livres illustrés de guerre pour enfants participaient de ce processus d'intériorisation. Les images en particulier, qui ont été reconnues comme une source historique à part entière avec leurs propres logiques et messages, sont dotées d'une force de persuasion. Elles participent, dans leur interaction avec le texte, au conditionnement des imaginaires et de la mémoire collective et ont un pouvoir d'action sur le spectateur ${ }^{8}$.

Pour traiter notre sujet, nous avons travaillé à partir d'un corpus représentatif de livres et de périodiques illustrés pour enfants et sur les programmes de deux maisons d'édition leaders sur le marché du livre de guerre pour enfants, Ferdinand Schreiber (Esslingen) et Josef Scholz (Mayence). Il est difficile d'estimer la diffusion de ces productions, dont les petits garçons dès l'âge de trois ou six ans, selon les supports, étaient les destinataires privilégiés. Leur mention fréquente dans des bibliographies critiques et des catalogues de bibliothèques scolaires donnent néanmoins à penser qu'ils connurent un succès conséquent.

Afin d'analyser la sortie de guerre de la littérature illustrée allemande et son impact potentiel, nous présenterons d'abord l'imaginaire héroïque qui constituait le fondement de la culture de guerre. Puis nous nous pencherons sur l'évolution des thèmes guerriers, en particulier à partir de l'année 1917 qui vit une nette baisse de la production d'ouvrages. Outre ces fluctuations, nous verrons ensuite que les éditeurs eurent tendance à surestimer le succès de leurs ouvrages. Enfin, nous examinerons la signification du silence qui marqua la littérature enfantine dès la fin 1918.

\section{La tradition de l'imaginaire héroïque de la guerre}

La tradition des illustrés et livres de guerre pour enfants était bien antérieure à 1914. Dans le Kaiserreich, les thèmes militaires et patriotiques connurent une augmentation aux alentours de 1913 en raison des commémorations du centenaire de la bataille des Nations de Leipzig et du jubilé d'argent de Guillaume II. Sujet de prédilection, les guerres napoléoniennes, stylisées en une guerre des nations - alors qu'elles restaient avant tout une guerre de cabinet classique - servaient à mettre en scène l'idéal d'une nation en armes ${ }^{9}$.

Issues de la peinture d'histoire officielle, les images tirées de ces imprimés reposaient sur de nombreux palimpsestes ${ }^{10}$. Leurs illustrateurs, tels que Michael Zeno Diemer, Angelo Jank et Willy Stöwer, étaient peintres d'histoire et uniformologues dont la fonction était de véhiculer une image traditionnelle de la guerre; des invariants iconographiques et thématiques - posture du chef de guerre sur sa monture, scènes de l'attaque de cavalerie et de l'assaut, mort violente du héros - entretenaient un imaginaire désuet et héroïque dont le pouvoir de persuasion était d'autant plus fort qu'il faisait appel à des réflexes émotionnels. Couplées à des poèmes romantiques, ces images visaient à renforcer le sentiment d'appartenance nationale des jeunes lecteurs.

\footnotetext{
${ }^{8}$ Christian Delporte / Laurent Gervereau / Denis Maréchal (dir.), Quelle est la place des images en histoire?, Paris, Nouveau Monde, 2008 ; Gerhard Paul, «Das Jahrhundert der Bilder. Die visuelle Geschichte und der Bildkanon des kulturellen Gedächtnisses », dans Id. (dir.), Das Jahrhundert der Bilder, vol. 1 : 1900 bis 1949, Göttingen, Vandenhoeck \& Ruprecht, 2009, p. 14-39.

${ }^{9}$ Voir par exemple les ouvrages et extraits d'illustrés suivants : Deutschland in Waffen, Stuttgart, Berlin, Deutsche Verlags-Anstalt, [1913], avec des illustrations de Michael Zeno Diemer, Franz Jüttner, Carl Röchling, Willy Stöwer, etc.

${ }^{10}$ Pour plus de précisions concernant cet aspect et pour consulter des illustrations, voir: Claire Aslangul / Bérénice Zunino, «Der Erste Weltkrieg in den Bildern : Heldentod oder Tod des Helden ? », dans Stéphane Pesnel (dir.), Der Erste Weltkrieg im Medium der Künste, actes du colloque du 13 décembre 2014 à la Maison Heinrich Heine, organisé par le Service culturel de l'Ambassade d'Allemagne, la Maison Heinrich Heine et l’université Paris-Sorbonne (EA3556 REIGENN) (à paraître chez Königshausen \& Neumann).
} 
Durant le conflit, après un creux de la production de livres entre août et novembre 1914 dû aux bouleversements de la mobilisation logistique et humaine, les thèmes martiaux, en nette augmentation par rapport à l'avant-guerre, continuèrent, parallèlement à l'introduction de nouveaux codes et figures (caricatures de l'ennemi, armes modernes), à être traités selon la même tradition iconographique ${ }^{11}$. La culture de guerre n'apparut donc pas ex nihilo dès août 1914 ; elle se développa sur le temps long, puisant dans des représentations apologétiques de la guerre issues de la peinture historique.

Bien avant le conflit, les enfants étaient bercés par une imagerie héroïque qui s'intensifia entre 1914 et 1918 pour prendre des accents triomphalistes. Par le prisme de ce bain visuel présent dans la littérature extrascolaire, mais aussi à l'école et dans les musées, les enfants, relégués au rang de victory watchers ${ }^{12}$, suivaient par procuration les «gestes » de leurs pères au front. Les imprimés leur inculquaient un modèle d'héroïsme masculin et alimentaient « leurs phantasmes d'une vie de soldats idéalisée » 13 . Eu égard à leur ancrage traditionnel et à leur puissance émotionnelle, nous pouvons aisément supposer que cet imaginaire héroïque influença durablement les représentations des jeunes gens.

La littérature illustrée ne laissait aucun doute quant à l'issue victorieuse du conflit. En 1917, elle connut toutefois une nette baisse, que nous nous proposons d'analyser dans notre deuxième partie.

\section{L'industrie du livre touchée par des difficultés matérielles à partir de 1917}

L'évolution des titres de guerre entre 1914 et 1918 donne à penser qu'il y eut une déprise de la guerre dès 1917 dans la littérature illustrée pour enfants. Après les premiers mois du conflit qui ralentirent la production, les deux premières années des hostilités se caractérisèrent par une activité éditoriale intense. Eu égard aux dates de parution des ouvrages de notre corpus, force est de constater que la période de fin 1914 à fin 1916 fut l'âge d'or du livre de guerre : par exemple, sur un total de quatre-vingt-douze livres d'images, quatre-vingt-deux furent édités entre 1914 et 1916. Seuls dix nouveaux ouvrages virent le jour entre 1917 et 1918, dont sept en 1917. L'échantillon représentatif de trente-deux récits et brochures patriotiques reflète le même phénomène : seuls trois ouvrages parurent entre 1917 et 1918. L'éditeur Schreiber mit son dernier livre patriotique sur le marché en 1917. La même année, Scholz n'édita que deux livres d'images, tous deux sans lien avec la guerre. L'année suivante, aucune nouvelle publication ne figurait à son programme ${ }^{14}$.

Tous domaines confondus, le marché éditorial connut une évolution similaire : alors que la production s'élevait à 22020 ouvrages en 1916, elle tomba à 14910 livres en 1917 pour atteindre 14743 en 1918 , soit le niveau de la production de $1880^{15}$. Quant aux livres consacrés à la guerre, 1099 nouvelles publications ont été répertoriées pour l'année 1915, contre 891 en 1916, 525 en 1917 et 513 en $1918^{16}$.

\footnotetext{
11 Voir par exemple: Schlachtenbilder. Malvorlagen für die Jugend, [Ravensbourg], [Maier], [1915] ; «Deutsche Husaren erobern eine französische Batterie », Der gute Kamerad, 1915, p. 401.

${ }^{12}$ Expression employée par Arndt Weinrich en référence aux travaux de Peter Merkl : Weinrich 2013, op. cit., p. 43.

«ihre Fantasien eines romantisierten Soldatenlebens», Robert Gerwarth, «Im "Spinnennetz". Gegenrevolutionäre Gewalt in den besiegten Staaten Mitteleuropas », dans Id. / John Horne (dir.), Krieg im Frieden. Paramilitärische Gewalt in Europa nach dem Ersten Weltkrieg, Göttingen, Wallstein, 2013, p. 108133, ici p. 116.

${ }^{14}$ Cornelia Schneider, «Die Bilderbuchproduktion der Verlage Jos. Scholz (Mainz) und Schaffstein (Köln) in den Jahren 1899 bis 1932 », université Johann Wolfgang-Goethe de Francfort-sur-le-Main, 1984.

${ }^{15}$ Barbara Kastner, «Statistik und Topographie des Verlagswesens », dans Georg Jäger (dir.), Geschichte des Deutschen Buchhandels im 19. und 20. Jahrhundert, vol. 1, partie 2 : Das Kaiserreich 1870-1918, MVB Marketing- und Verlagsservice des Buchhandels GmbH, Francfort/Main, 2003, p. 300-367.

${ }^{16}$ Thomas F. Schneider / Julia Heinemann / Frank Hischer / Johanna Kuhlmann / Peter Puls, Die Autoren und Bücher der deutschsprachigen Literatur zum Ersten Weltkrieg 1914-1939. Ein bio-bibliographisches Handbuch, Göttingen, Vandenhoeck \& Ruprecht, 2008.
} 
La cherté et la pénurie des matières premières, couplées au manque de main-d'œuvre qualifiée, entravaient la production. Les difficultés d'approvisionnement en huiles, en vernis et en couleurs portaient atteinte au fonctionnement des machines et à la conception des ouvrages. Remplacées par des produits (ersatz) de qualité médiocre, ces substances donnaient des résultats moins satisfaisants. La pénurie de papier se fit le plus gravement sentir; il fut rationné à partir d'avril $1916^{17}$. Les imprimeurs et les éditeurs n'obtinrent généralement que $60 \%$ de leur consommation habituelle. Bien que son accès aux matières premières fût vraisemblablement facilité en raison de son statut de branche importante pour l'industrie de guerre, Schreiber, qui éditait aussi des ouvrages pour le front, s'inquiétait en janvier 1918 de ses stocks de papier, de couleurs et de colle ${ }^{18}$.

Cette pénurie engendra une augmentation fulgurante des prix. Entre début 1916 et avril 1918 , les albums ne connurent pas moins de six augmentations (de $5 \%$ à $50 \%$ ) : un livre qui coûtait trois à cinq marks avant 1914 pouvait atteindre 11,50 marks en avril 1918, soit près de trois fois le prix de vente moyen de $1913^{19}$. À partir de juillet 1917 , le prix indiqué dans les brochures publicitaires des éditeurs n'était plus garanti.

Cette déprise de la guerre ne fut néanmoins pas équivalente à une démobilisation culturelle précoce. Face à une telle pénurie de nouveaux ouvrages, les pédagogues continuèrent à recommander, dans la plupart des revues pédagogiques et des catalogues et bibliographies critiques, les livres édités durant la première moitié du conflit ${ }^{20}$. En d'autres termes, les années 1917-1918 se caractérisaient par un fort décalage entre une nette baisse de la production et une poursuite indirecte de la mobilisation par le biais d'ouvrages parus entre 1914 et 1916.

Durant les dernières années du conflit, les catalogues et bibliographies critiques jouèrent donc un rôle central de relais de la mobilisation littéraire. Selon nous, la persistance des titres de guerre dans ces productions était due aux efforts de ces multiplicateurs qui tentaient d'entretenir l'adhésion populaire et d'afficher leur propre patriotisme. La recommandation des livres de guerre faisait partie des devoirs qu'on leur attribuait. Dans une société entièrement tournée vers la guerre et mue par la foi en la victoire, comment auraient-ils pu renoncer du jour au lendemain à recommander ces ouvrages ? Ces pédagogues et critiques littéraires n'échappèrent pas à la pression sociale ni aux attentes collectives ${ }^{21}$ : le patriotisme était de mise, le défaitisme fermement condamné.

En 1917, les thèmes guerriers étaient néanmoins en perte de vitesse. Les indications collectées concernant les tirages et les éventuels invendus confirment cette hypothèse.

\section{Succès des livres de guerre surestimé par les éditeurs}

Cette poursuite indirecte de la mobilisation littéraire par les pédagogues s'accompagna d' « un affaiblissement constant de la demande de best-sellers de guerre ${ }^{22}$ au profit de livres sans lien avec les hostilités, d'après le bilan de Karl Scholz pour l'année 1918. Les résultats des ventes de livres de guerre pour enfants furent modérés par rapport aux attentes des contemporains, en particulier des associations patriotiques qui éditèrent certains ouvrages. Sur

\footnotetext{
17 Karl Scholz, «Die volkswirtschaftliche Bedeutung des deutschen Bilderbuchverlags », université Johann Wolfgang-Goethe de Francfort-sur-le-Main, 1922 ; Siegfried Lokatis, « Der militarisierte Buchhandel im Ersten Weltkrieg », dans Georg Jäger (dir.), Geschichte des Deutschen Buchhandels im 19. und 20. Jahrhundert, vol. 1, partie 3 : Das Kaiserreich 1870-1918, de Gruyter, Berlin, 2010, p. 444-469.

${ }^{18}$ Wirtschaftsarchiv Baden Württemberg (WABW), fonds Ferdinand Schreiber : B91 Bü771, non paginé.

${ }^{19}$ Frédéric Barbier, L'empire du livre. Le livre imprimé et la construction de l'Allemagne contemporaine (18151914), Paris, Le Cerf, 1995, p. 67.

${ }^{20}$ Voir par exemple : Joseph Antz / Thalhofer, «Jugendschriften », Literarischer Ratgeber für die Katholiken Deutschlands, 1915, p. 42-43.

${ }^{21}$ Cédric Marty, «"À la baïonnette !" Approche des imaginaires à l'épreuve de la guerre 1914-1918 », thèse de doctorat, Rémy Cazals, université Jean Jaurès de Toulouse, 2014.

22 «ein stetiges Nachlassen der Nachfrage nach 'Kriegsschlagern' », Scholz 1922, op. cit., p. 80.
} 
les 40000 exemplaires de Michel Hannemanns Traum $^{23}$ parus en 1916, 16500 restaient encore à disposition de l'association du Vaterländischer Frauenverein l'année suivante. ${ }^{24}$ Par rapport au nombre moyen d'exemplaires produits à l'époque par les maisons d'édition, ces chiffres sont considérables. Plus de la moitié avait été écoulée en un laps de temps relativement court, mais, en 1917, une quantité non négligeable de livres restait en stock. Eu égard aux conditions de vie et au moral de la population durant les dernières années du conflit, nous doutons fortement qu'elle ait pu être écoulée avec le succès escompté par les éditeurs. D'autres entreprises se soldèrent aussi par des échecs relatifs : sur les 4000 exemplaires de Des Kindes Kriegsbilderbuch ${ }^{25}$ sept cents, soit près du quart, étaient encore invendus au 27 mars 1918, alors que la Croix-Rouge projetait à l'origine d'en produire 10000 exemplaires. ${ }^{26}$ La somme de 2144 marks qui revint à cette organisation devait servir au soin des blessés et des malades, notamment à la formation du personnel hospitalier. Malgré les cinq autorisations de prolongation accordées jusqu'en 1918, le succès de cet ouvrage, non négligeable, fut visiblement inférieur aux attentes de ses éditeurs.

À une époque où la lassitude s'accroissait et où les autorités, en pleine crise de légitimité, peinaient à répondre aux besoins vitaux de la population, ces initiatives encouragées par des associations patriotiques, en dépit de leur vocation universelle, correspondaient à une tentative, dérisoire, de stabiliser l'arrière et de consolider la monarchie. La guerre ne faisait manifestement plus autant vendre. La population devait faire face à l'épuisement, aux privations et à la sous-nutrition. Vraisemblablement sous l'effet de la faim et du découragement, Elise Nollenberger (née en 1903 à Kirchheim am Neckar) interrompit brutalement son journal intime à cette période ${ }^{27}$. Le nombre d'entrées du cahier d'Elfriede Kuhr (née en 1902 en Prusse-Orientale) avait sensiblement baissé dès $1916^{28}$. Pour un nombre croissant de familles issues des classes moyennes et supérieures, qui constituaient le lectorat principal $^{29}$, l'achat de livres, tous domaines confondus, devenait un luxe. La chute de la production d'ouvrages intervint peu de temps après que les conséquences matérielles et domestiques des hostilités s'étaient fait sentir sur ces catégories sociales. Au début du conflit, les classes moyennes et supérieures avaient pu puiser dans leurs économies et recourir à l'aide de proches pour subvenir à leurs besoins. Bien que moins rapidement affectées, ces classes sociales étaient désormais les catégories les plus gravement touchées par la guerre. Le pouvoir d'achat de la bourgeoisie cultivée, des fonctionnaires, des employés et des artisans s'effondra ${ }^{30}$.

Outre la production des albums et des récits de guerre pour la jeunesse qui chuta à partir de 1917, les thèmes patriotiques connurent un infléchissement dans les revues à la même

\footnotetext{
${ }^{23}$ Richard Bars / Moritz Pathe, Michel Hannemanns Traum. Ein Buch für Deutschlands Jugend, éd. par le Vaterländischer Frauenverein, Berlin-Charlottenbourg, Märkische Verlagsanstalt, [1916].

${ }^{24}$ Geheimes Staatsarchiv Preußischer Kulturbesitz (GStA PK), I HA Rep. 191 Ministerium für Volkswohlfahrt. Staatskommissar für die Regelung der Wohlfahrtspflege, $\mathrm{n}^{\circ} 3127$, lettre datée du 2 octobre 1917 du Vaterländischer Frauenverein (section Berlin-Charlottenburg) au commissariat de police royal de Charlottenburg, non paginé.

${ }^{25}$ Bernhard Wende, Des Kindes Kriegsbilderbuch, éd. par le Comité central des associations allemandes de la Croix-Rouge, Berlin-Schöneberg, Oestergaard, [1915].

${ }^{26}$ GStA PK, I HA Rep. 191 Nr. 3050, correspondance entre le comité central de la Croix-Rouge allemande et le commissaire chargé du contrôle de l'action caritative, du 2 octobre 1915 au 27 mars 1918, non paginé.

27 Peter Knoch, «Kinder im Krieg 1914-18. Zwei Mädchen schreiben Kriegstagebuch », dans Gerhard Hergenröder / Eberhard Sieber (dir.), Varia historica. Beiträge zur Landeskunde und Geschichtsdidaktik, Rainer Jooß zum 50., Plochingen, Herba, 1988, p. 443-488.

28 Jo Mihaly (Elfriede Kuhr), ...da gibt's ein Wiedersehen! Kriegstagebuch eines Mädchens 1914-1918, Fribourg, Kerle Verlag, 1982.

${ }^{29}$ Bérénice Zunino, «La littérature illustrée pour enfants à l'époque de la Première Guerre mondiale. Origines et évolution de la culture de guerre enfantine allemande », thèse de doctorat, Jean-Paul Cahn / Oliver Janz, université Paris IV-Sorbonne / Freie Universität Berlin, 2014.

30 Hans-Ulrich Wehler, Deutsche Gesellschaftsgeschichte, vol. 4: 1914-1949, vom Beginn des Ersten Weltkrieges bis zur Gründung der beiden deutschen Staaten, Munich, Beck, 2003, p. 69-111.
} 
période ; à partir de la fin 1916, la revue de confession protestante Deutscher Kinderfreund ${ }^{31}$ se caractérisait par un ton plus sobre : les privations, la mobilisation matérielle des enfants et, sporadiquement, les blessures et les amputations des soldats, y occupaient une place croissante. Une évolution des illustrations vers plus de sobriété accompagna ces infléchissements : les caricatures disparurent au profit de scènes de genre, de silhouettes, mais aussi de dessins dont les références au conflit étaient de plus en plus discrètes.

Autrement dit, l'effervescence de la mobilisation littéraire des enfants correspondit à la première phase du conflit, jusqu'en 1916. En 1917, la production s'effondra. Après les échecs des offensives du printemps 1918 et la retraite des troupes, le moral de l'opinion publique allemande chuta définitivement en octobre ${ }^{32}$. Personne ne croyait plus en la victoire finale martelée par les autorités et la presse. De la littérature enfantine se dégagea dans l'immédiat après-guerre un silence éloquent.

\section{Silence de l'après-guerre, une mémoire traumatique du conflit}

Hormis de rares livres de guerre pour enfants qui continuèrent après la défaite à dépeindre le conflit sur un ton héroïque, les hostilités tombèrent bientôt dans l'oubli. Dans les numéros de septembre-octobre 1918, presque du jour au lendemain, alors que l'armée allemande se trouvait « en état de mort clinique ${ }^{33}$ et que le pays commençait à être secoué par la révolte des marins, la plupart des revues mensuelles, bimensuelles ou hebdomadaires (comme Hänsel und Gretel, Der gute Kamerad, Deutscher Kinderfreund) n'abordèrent plus le conflit. Il n'y eut aucune mention de l'armistice. La fin du conflit vit aussi l'arrêt complet de la production d'albums patriotiques. Il y eut également des projets avortés : la deuxième partie de l'ouvrage humoristique Das Weltkriegesbilderbuch qui devait paraître, selon la préface optimiste du premier volume ${ }^{34}$, après la victoire, ne vit jamais le jour. Bien que les indications de la bibliographie nationale soient partielles, elles donnent une idée approximative de l'état du marché durant l'immédiat après-guerre : entre 1921 et 1930, seulement seize livres de guerre furent répertoriés sur un total de cent-soixante-et-onze titres ${ }^{35}$. Notons par ailleurs que la quasi-disparition des sujets guerriers dans les livres pour enfants s'inscrivait à contre-courant des tendances de la littérature pour adultes qui allait continuer de traiter abondamment du conflit dans l'entre-deux-guerres ${ }^{36}$.

Ce silence tint néanmoins une place essentielle dans toute la société allemande d'aprèsguerre. Il révèle selon nous « une mémoire empêchée », « de type traumatique », ${ }^{37}$ un refus de se confronter à la défaite. Nombreux furent les Allemands qui eurent l'impression de n'avoir pas vraiment perdu la guerre, d'autant plus que le retrait des troupes s'était effectué sans percée spectaculaire alliée ni avancée sur le sol allemand. Aux yeux de la population, confrontée à l'absurdité de ses sacrifices durant plus de quatre années de guerre et à la poursuite du blocus, ressentie comme une injustice, la défaite était d'autant plus inacceptable que la guerre avait été présentée comme juste. Imposée par le camp ennemi, cette paix ne

\footnotetext{
${ }^{31}$ Deutscher Kinderfreund, Ninck, 1916.

${ }^{32}$ Wilhelm Deist, «Le "moral" des troupes allemandes sur le front occidental à la fin de l'année 1916 », dans Jean-Jacques Becker / Jay Winter / Gerd Krumeich / Annette Becker (dir.), Guerre et cultures (1914-1918), Paris, Colin, 1994, p. 91-102.

${ }^{33}$ Pierre Jardin, Aux racines du mal. 1918, le déni de la défaite, Paris, Tallandier, 2005, p. 12.

${ }^{34}$ Ludwig Pronold / Albert Henselmann, Das Weltkriegsbilderbuch. Franzl-Michl - Mohammed, Straubing, Munich, Attenkofer, 1916.

${ }^{35}$ Eberhard Demm, «Deutschlands Kinder im Ersten Weltkrieg: Zwischen Propaganda und Sozialfürsorge », Militärgeschichtliche Zeitschrift, ${ }^{\circ}$ 60, 2001, p. 51-98.

${ }^{36}$ Wolfgang Natter, Literature at War, 1914-1940. Representing the "Time of Greatness" in Germany, New Haven, Londres, Yale University Press, 1999.

${ }^{37}$ Étienne François, «L'histoire et la mémoire. L’importance des discours sur le passé », dans Reiner Marcowitz / Werner Paravicini (dir.), Vergeben und vergessen? Vergangenheitsdiskurse nach Besatzung, Bürgerkrieg und Revolution. Pardonner et oublier? Les discours sur le passé après l'occupation, la guerre civile et la révolution, Munich, Oldenbourg, 2009, p. 17-26, ici p. 24.
} 
pouvait être qu'illégitime. Il en allait de même des manuels scolaires utilisés sous la République de Weimar : parfois, la fin de la guerre y était simplement ignorée ; les manuels s'achevaient par la paix victorieuse de Brest-Litovsk ou sur les offensives réussies du printemps 1918. Parallèlement, malgré les efforts des autorités pour rompre avec la tradition monarchique, une partie des ouvrages parue en période de guerre resta en circulation ${ }^{38}$.

Couplée à la tradition ancienne des représentations apologétiques de la guerre, cette absence apparente de réaction dans la littérature illustrée comme à l'école accentua probablement l'incompréhension des enfants et leurs difficultés à accepter la défaite. Wolfgang Yourgrau (né en 1908 à Kattowitz) en a témoigné :

Je n'avais jamais entendu dire que nous avions perdu la guerre, ni à l'école ni à la maison. [...] Je ne comprenais rien à tout ce tumulte, je répétais les propos des adultes quoi qu'ils racontassent, et regrettais le passé, l'empereur, la guerre, les parades et les cérémonies ${ }^{39}$.

Sebastian Haffner (né en 1907 à Berlin) vécut lui aussi la défaite comme un choc :

À quoi comparer ce que je ressentis - ce que ressent un garçon de onze ans qui voit s'écrouler tout son monde imaginaire ${ }^{40}$ ?

Comment, après avoir été bercés dès leur plus tendre enfance par un imaginaire héroïque, malgré les infléchissements et la lassitude de 1917, ces enfants auraient-ils pu admettre le revirement de la fin de l'année 1918 ? Cette déception engendra une dévalorisation des figures masculines et compliqua indirectement la démobilisation culturelle de cette cohorte qui représenta aussi un réservoir important pour le mouvement national-socialiste. Les corps francs et les milices paramilitaires, qui regroupaient à eux seuls 250000 à 400000 membres, parmi lesquels la Kriegsjugendgeneration occupait une place prépondérante, ainsi que la bündische Jugend permirent à nombre de jeunes garçons, à l'instar d'Ernst von Salomon, de se démarquer de la génération des pères ${ }^{41}$.

En conclusion, nous avons tenté dans cette communication d'intégrer les livres de guerre pour enfants au champ de l'histoire culturelle pour approfondir les réflexions sur les liens entre la littérature patriotique pour enfants et la difficile démobilisation culturelle de l'Allemagne au sortir de la Première Guerre mondiale. Couplé au silence traumatique de l'après-1918, l'imaginaire héroïque de la guerre qui avait constitué le fondement de la culture de guerre dès l'avant-1914 marqua sans doute durablement les représentations mentales des jeunes générations et leurs attentes envers l'issue - nécessairement victorieuse - du conflit, compliquant la démobilisation des esprits. Toutefois, la mobilisation culturelle des enfants ne fut pas un processus linéaire : si elle fut intense entre la fin 1914 et l'année 1916, il ressort clairement de nos recherches que les ouvrages patriotiques connurent une nette baisse dès 1917. Par ailleurs, les indications concernant les tirages et le nombre d'invendus montrent que leur succès, indéniable, fut néanmoins surestimé par les acteurs participant à la chaîne de production et de diffusion, éditeurs et associations patriotiques.

Durant l'après-guerre qui devait être un vaste champ de mémoires concurrentes du conflit, des pacifistes jugèrent ces ouvrages patriotiques assez pernicieux pour se lancer dans un combat contre cette culture de guerre. Dans Les derniers jours de l'humanité (1922) Karl Kraus, fervent collectionneur de cartes postales, d'affiches, de photographies et de livres patriotiques entre 1914 et 1918, vilipenda les jouets, jeux et livres de guerre tant allemands

\footnotetext{
${ }^{38}$ Pierre Jardin, «La légende du "coup de poignard" dans les manuels scolaires allemands des années 1920 », Vingtième Siècle. Revue d'histoire, $\mathrm{n}^{\circ}$ 41, janvier-mars 1994, p. 39-50.

39 «Ich hatte nie gehört, dass wir den Krieg verloren hatten, nicht in der Schule, nicht in meiner Familie. [...] Ich verstand von all dem Trubel nichts, redete den Erwachsenen nach, was immer sie auch erzählten, und sehnte die alte Zeit wieder herbei, den Kaiser, den Krieg, die Paraden, die Feiern. » Ursula Blömer / Detlef Garz (éd.), ,, Wir Kinder hatten ein herrliches Leben... " Jüdische Kindheit und Jugend im Kaiserreich, Oldenbourg, Bis-Verlag, 2000, p. 245.

${ }^{40}$ Sebastian Haffner, Histoire d'un Allemand. Souvenirs (1914-1933), Arles, Actes Sud, 2004 (1 éd. 2000), p. $48-49$.

${ }^{41}$ Robert Gerwarth, «Im "Spinnennetz". », art. cit.
} 
qu'autrichiens. ${ }^{42}$ Sur un ton véhément et images à l'appui, Ernst Friedrich, dans son ouvrage paru pour le dixième anniversaire du début du conflit, Krieg dem Kriege!, dénonça les soldats de papier, les jouets et les livres de guerre comme l'étape préliminaire d'une éducation «au plus grand des crimes : l'homicide $\gg$.

${ }^{42}$ Karl Kraus, Die letzten Tage der Menschheit. Tragödie in fünf Akten mit Vorspiel und Epilog, acte IV, scène 22, Francfort/Main, Suhrkamp, 1986 ( $1^{\mathrm{e}}$ éd. 1922), en particulier p. 463.

43 «[Erziehung] zum größten Verbrechen, zum Menschenmord », dans Ernst Friedrich, Krieg dem Kriege!, Munich, Deutsche Verlagsanstalt, 2004 ( $1^{\mathrm{e}}$ éd. 1924), p. 42. 